\title{
A CW normal-conductive RF gun for free electron laser and energy recovery linac applications
}

\author{
K. Baptiste ${ }^{\mathrm{a}}$, J. Corlett ${ }^{\mathrm{a}}$, S. Kwiatkowski ${ }^{\mathrm{a}}$, S. Lidia ${ }^{\mathrm{a}}$, J. Qiang ${ }^{\mathrm{a}}$, F. Sannibale ${ }^{\mathrm{a}, *}, \mathrm{~K}_{\text {. Sonnad }}$, J. Staples ${ }^{\mathrm{a}}$, S. Virostek ${ }^{\mathrm{a}}$, \\ R. Wells ${ }^{\mathrm{a}}$ \\ ${ }^{a}$ Lawrence Berkeley National Laboratory, One Cyclotron Road, Berkeley, California 94720, US
}

\begin{abstract}
Currently proposed energy recovery linac and high average power free electron laser projects require electron beam sources that can generate up to $\sim 1 \mathrm{nC}$ bunch charges with less than $1 \mathrm{~mm} \mathrm{mrad} \mathrm{normalized} \mathrm{emittance} \mathrm{at} \mathrm{high} \mathrm{repetition}$ rates (greater than $\sim 1 \mathrm{MHz}$ ). Proposed sources are based around either high voltage DC or microwave RF guns, each with its particular set of technological limits and system complications. We propose an approach for a gun fully based on mature RF and mechanical technology that greatly diminishes many of such complications. The concepts for such a source as well as the present RF and mechanical design are described. Simulations that demonstrate the beam quality preservation and transport capability of an injector scheme based on such a gun are also presented.
\end{abstract}

Key words: electron source, low emittance, high brightness, ERL-FEL Injector

PACS: 29.25.Bx, 29.27.Bd, 29.27.Eg, 41.60.Cr

\section{Introduction}

A number of proposed projects in energy recovery linacs (ERL) and linac-based soft-xray-VUV free electron lasers (FEL) requires injection of $100 \mathrm{pC}-1 \mathrm{nC}$ electron bunches at repetition rates from $\mathrm{kHz}$ to hundreds of MHz [1, 2, 3, 4, 5, 6, 7]. For both ERL and FEL applications, production of high-brightness electron beams is critical in achieving the desired $\mathrm{x}$-ray performance and normalized beam emittances lower than $1 \mathrm{~mm} \mathrm{mrad}$ are required. This places particular burdens on the cathode, gun and injector systems. Limiting early emittance growth requires minimum accelerating electric fields in the gun of $\sim 20 \mathrm{MV} / \mathrm{m}$ and gun voltages of $\sim 500 \mathrm{kV}$ or higher [8]. Obtaining these fields and voltages in a DC gun requires a significant effort and expertise, especially in preventing insulator breakdown. Voltages of up to $\sim 350 \mathrm{kV}$ have been already demonstrated [9] and there is a group trying to extend such a value up to $\sim 750 \mathrm{kV}[10]$.

Superconducting, high-frequency cavities are likely candidates for high repetition rate photoinjectors, and an intense $R \& D$ activity around the world is presently

\footnotetext{
${ }^{*}$ Corresponding author. Tel: +1 510486 5924; Fax: +1 510486 4960

Email addresses: fsannibale@lbl.gov (F. Sannibale)

Preprint submitted to Nuclear Instruments and Methods A
}

directed to demonstrate the capability of such a scheme to operate with the required performances [11, 12]. Superconducting technology for guns is not yet mature and additionally is not compatible with many cathode materials. Moreover, it prevents by flux exclusion (Meissner effect) the application of controlled magnetic fields at the cathode for emittance manipulation techniques [13, 14]. High-frequency (L- to S-band) normalconducting radio-frequency (RF) guns do not present such limitations; however, the average power density in the cavity structure limits the practical repetition rate to the $\mathrm{kHz}$ range [15]. By decreasing the RF frequency, the size of the cavities increases with a beneficial reduction of the power density on the structure walls. In this situation, the repetition rate of the system can increase and continuous wave $(\mathrm{CW})$ operation can be achieved. The Boeing gun has achieved 25\% duty cycle operation at $433 \mathrm{MHz}$ [16], a $700 \mathrm{MHz} \mathrm{CW}$ normal conducting gun has been proposed [17], and a group at Los Alamos is completing the construction of a $700 \mathrm{MHz}$ normalconducting RF gun where a sophisticated and state of the art cooling system has been designed to operate in CW mode $[18,19]$.

In our approach [20, 21, 22], based on an idea of $\mathbf{J}$. Staples and developed at the Lawrence Berkeley National Laboratory in the framework of the activities

March 5, 2009 
on next generation light sources $[23,1]$, we lower the frequency further down into the Very High Frequency (VHF) range (30-300 MHz) adopting a design with a cavity operating at $\sim 100 \mathrm{MHz}$. The resulting modest power load on the walls is compatible with the use of conventional technology for cooling the structure and makes it capable to operate in $\mathrm{CW}$ mode at $750 \mathrm{kV}$ across the accelerating gap with a $20 \mathrm{MV} / \mathrm{m}$ gradient. The relatively large size of the cavity and the long RF wavelength allow the design of an efficient vacuum system with large pumping apertures capable of pressures compatible with the operation of many kind of cathode materials including semiconductors. An imbedded solenoid permits the easy control of the magnetic field in the cathode area. The choice of the frequency right in the middle of the FM broadcast band ensures the availability of commercial RF power sources. Many experiments utilizing pump-probe techniques require pulses to arrive at repetition rates in the $1 \mathrm{kHz}-1 \mathrm{MHz}$ range. Our $\mathrm{CW}$ VHF-gun combined with the use of high quantum efficiency cathodes and relatively modest power laser systems can be, therefore, used as the main component for the injector of an FEL to readily serve this extremely active experimental community.

A similar concept that has been proven in operations is the VHF gun used at the ELSA $19 \mathrm{MeV}$ linac $[24,25,26]$, in which the $144 \mathrm{MHz}$ gun has produced high charge-low emittance beams within a 150 $\mu$ s macropulse at $10 \mathrm{~Hz}$ repetition rate.

In the next sections, we describe the design concept of the VHF gunand the technical design solutions adopted. We also provide an example of a beam dynamics simulation showing the capability of such an injector to preserve the beam quality as required for FEL or ERL operation.

\section{The VHF RF Gun System}

The overall design of the gun is dictated by requirements of beam dynamics to produce the desired electron beam parameters, maximizing the shunt impedance to minimize the RF power requirement, minimizing the power density on the walls, allowing access for water cooling passages near high power density regions, minimizing field emission and multipactoring, and allowing for high conductance vacuum pumping.

Beam dynamics issues will be treated in section 3 . Here we concentrate our attention on the engineering questions related to the design of the VHF gun. But before doing that, we note that the $\sim 10 \mathrm{~ns}$ RF period is a relatively long time when compared with either the typical (few tens of ps) bunch length or the few hundreds of

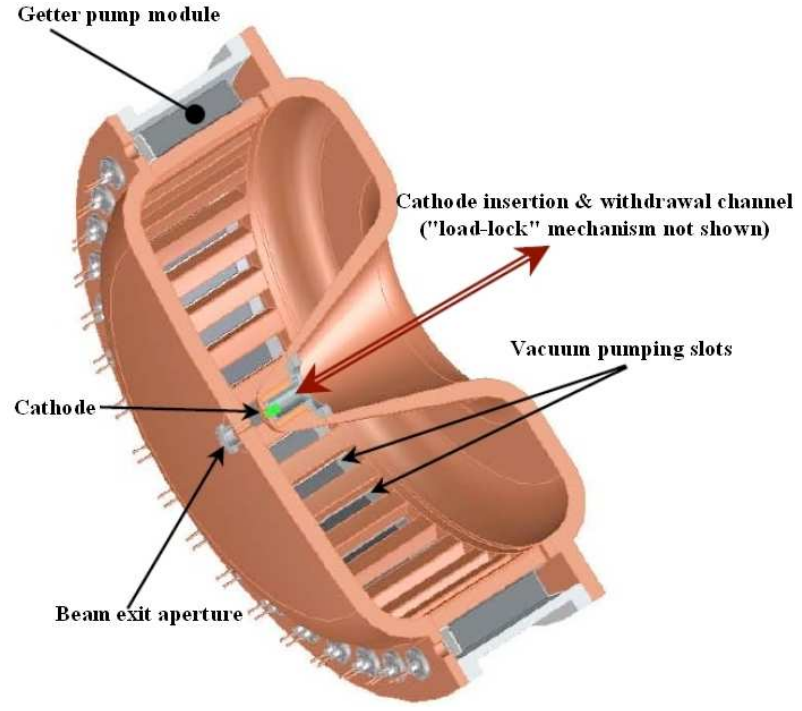

Figure 1:

ps beam transit time through the cavity gap. This makes the beam dynamics of the VHF gun similar to that of a DC gun. Extensive simulations [8] showed that the required beam performance can be obtained with DC fields of $\sim 20 \mathrm{MV} / \mathrm{m}$ and with energy out of the gun greater than $\sim 500 \mathrm{kV}$. In our design we exploit these results and assume $20 \mathrm{MV} / \mathrm{m}$ and $750 \mathrm{kV}$ as the nominal values for the accelerating field and for the final energy respectively.

Figure 1 shows a 3-D view of the RF structure, while Table 1 contains the cavity main parameters. The normal-conducting structure will be fabricated from copper-plated steel and the re-entrant geometry allows for the desired resonant frequency while keeping the size of the whole structure reasonably small. The steel structure presents a better strength to weight ratio and is less expensive respect to the solid copper option, and the copper plated surface can achieve RF and vacuum performances at the same level of the solid copper case when the proper technology is used [28, 29].

The cavity geometry was carefully optimized to maximize the shunt impedance, to minimize the wall power density, to reduce the mechanical stress, simplify fabrication and facilitate photocathode replacement. At the nominal quality factor $Q_{0}=37800$, the cavity requires an RF power of $73 \mathrm{~kW}$ for an accelerating gradient of $20 \mathrm{MV} / \mathrm{m}$ at the cathode and $\sim 17.5 \mathrm{MV} / \mathrm{m}$ average over a gap of $4 \mathrm{~cm}$ for a final beam voltage of $750 \mathrm{kV}$. The modest $8 \mathrm{~W} / \mathrm{cm}^{2}$ maximum power density on the cavity walls is readily dissipated with conventional water 


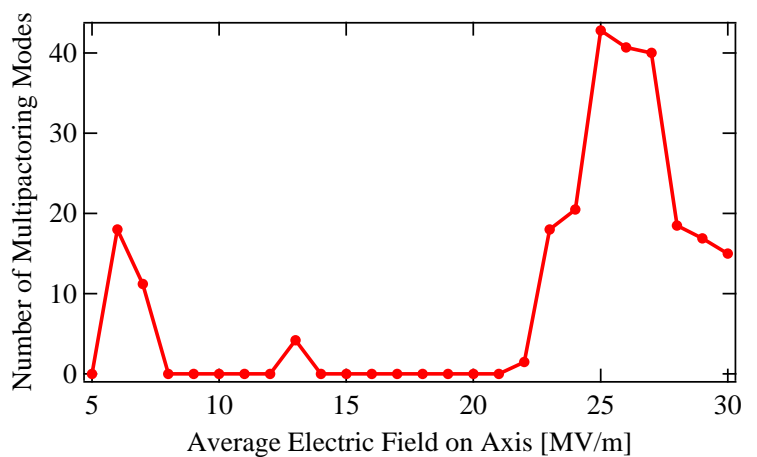

Figure 2:

cooling systems.

To provide $20 \mathrm{MV} / \mathrm{m}$ at the cathode, the maximum surface electric field at any point in the cavity is approximately $26 \mathrm{MV} / \mathrm{m}$. Experience with existing RF structures suggests that such low fields should induce negligible field emission after conditioning if the proper construction techniques, materials and tolerances are used.

The maximum electric field of $26 \mathrm{MV} / \mathrm{m}$ should not represent difficulties with respect to voltage breakdown. For comparison, the similar frequency $144 \mathrm{MHz}$ ELSA injector has been routinely operated with a $150 \mu$ s pulse at $33 \mathrm{MV} / \mathrm{m}$ peak field with no serious breakdown [25], and in more recent times, the same injector successfully operated at $25 \%$ higher values $(41 \mathrm{MV} / \mathrm{m})$ [27].

To operate at the nominal voltage of $750 \mathrm{MV}$, a stored energy in the RF structure of $4.1 \mathrm{~J}$ is required. This value does not represent a concern for cavity wall damage even in the case of severe voltage breakdowns in which the energy can be entirely released during an arc.

Multipactoring is a complex phenomenon. For any frequency choice there are always RF power levels that excite potentially dangerous multipactoring resonances. An analysis of the phenomenon for our VHF structure has been performed by using two independent codes (Fishpact and Analyst ${ }^{T M}[30,31]$ ) and indicates that there are no multipactoring modes in the region around the expected operating voltage. Figure 2 shows a summary of the Fishpact calculations. Two multipactoring regions exists at two low field levels, corresponding to 6 and $13 \mathrm{MV} / \mathrm{m}$, and more significant at levels above 22 $\mathrm{MV} / \mathrm{m}$. The region from 14 to $21 \mathrm{MV} / \mathrm{m}$ does not show resonances, in particular at the field of $18.75 \mathrm{MV} / \mathrm{m}$ corresponding to our nominal gap voltage of $750 \mathrm{kV}$ [32].

An additional advantage of VHF structures with respect to their higher frequency counterparts is in the geometry of the RF power input coupler. For the VHF

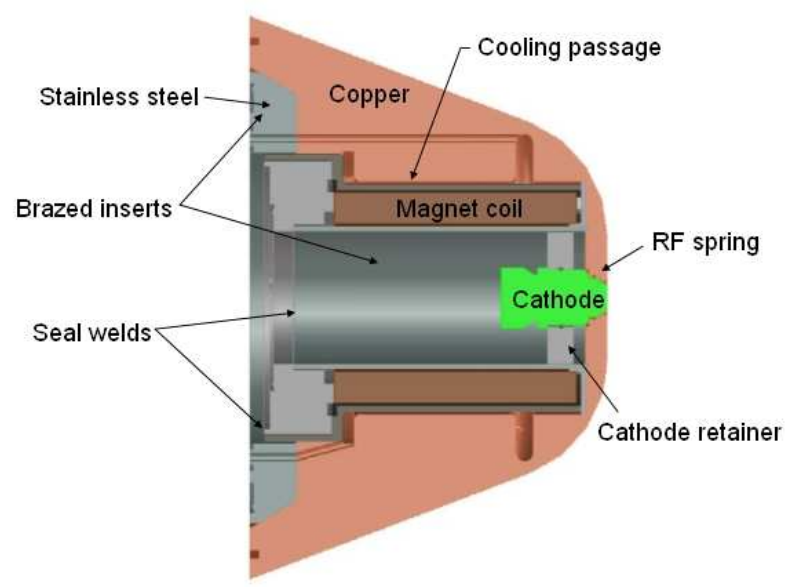

Figure 3:

case, a simple scheme with one or more loops can be used to feed the RF power into the structure. Loops introduce minimal distortion of the cavity fields (important from the beam dynamics point of view), and are easy to design, fabricate, and tune. For frequencies higher than VHF, the loop size decreases and the power dissipation in the loop can become too large. Ultimately a coupling iris must be used for higher frequencies with a consequent increase in design complexity and field distortion. For the case of the $106 \mathrm{MHz}$ structure, one or two drive loops will provide sufficient RF power.

Figure 3 shows the detail of the VHF gun cathode area designed to operate with a load-lock mechanism (for easy, in-vacuum replacement of photocathodes) based on the FLASH gun design [33, 34]. A solenoidal coil is embedded in the 'nose' of the cavity and can be used to either null the on-axis magnetic flux, or to provide up to $\sim 0.05 \mathrm{~T}$ at the cathode plane to create correlations in the emitted beam phase space that could be required by emittance exchange techniques $[14,13]$.

The VHF cavity has a relatively large volume to pump out; however, the long wavelength allows for large vacuum ports and consequently for better pumping speed. Design of 36 pumping slots, $4.9 \mathrm{~cm}$ wide separated by bars also $4.9 \mathrm{~cm}$ wide, around the cavity equator have been assessed; their impact on RF performance is negligible [35, 36]. The frequency shift due to the presence of the slots is very small. Likewise, the increase in the RF power required is also small. Figure 4 shows a MAFIA code [37] simulation illustrating the logarithm of the magnitude of the electric field in the outer pump slot region of cavity expressed in $\mathrm{dB}$. The attenuation of the field along the depth of 


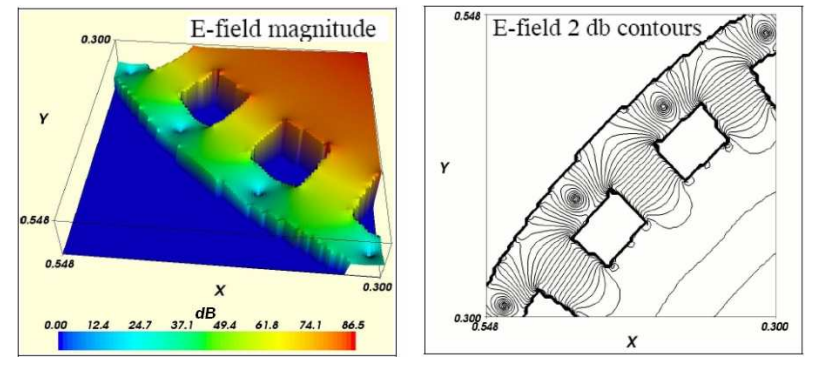

Figure 4:

the slot is seen to be about 30 contours, or $60 \mathrm{~dB}$, consistent with a waveguide-beyond-cutoff. Calculation of the magnetic field (not shown) gives identical results in the slots. There is a local minimum in the field magnitude at points directly behind the bars, which are a good spot for getter pumps to be located. The RF power absorbed by a getter pump module in this position has been estimated to be $\sim 1 \mathrm{~W}$. The large anode wall is slightly curved to provide greater stiffness against deflection under vacuum. An all-metal structure is suitable for bake-out procedures, and the large outer diameter provides excellent accessibility for the getter pumps. Initial calculations using the SAES getter wafer modules WP1250/2 [38] have shown that the cavity should support a vacuum down to the high $10^{-9} \mathrm{~Pa}\left(10^{-11}\right.$ Torr $)$ range. Depending on the fabrication technique, some of the getter modules can potentially generate microparticles (dust) during operation. Such a situation could trigger voltage breakdown in the cavity and must be avoided. The final decision on the getter module to be used will be made only after a careful investigation of the issue.

The VHF frequency cavity is larger than the $\mathrm{S}$ and $\mathrm{L}$ band counterparts and the longer RF wavelength relaxes the mechanical tolerance and surface roughness requirements. Thermal and structural analysis of the cavity has been performed as well. Surface heat loads derived from the SUPERFISH code [39] were used as input to the ANSYS FEA code [40] and applied to an accurate CAD model of one quadrant of the gun cavity. Figure 5 shows a contained maximum temperature increase of $\sim 83{ }^{\circ} \mathrm{C}$ in the center of the central cone, and a much smaller increase at the cathode area. This temperature variation does not represent a concern from either the mechanical or the RF point of view. The associated mechanical stress is quite small and the cavity frequency at thermal equilibrium will be regulated by the tuning system described later in this section. Cooling is provided by water passages in machined struc- tures brazed to the exterior of the cavity in high heat load regions and by brazed-on cooling tubes in the low heat regions. The greatest temperature rise experienced at the center of the central cone of the cavity, is due primarily to the increased thickness of steel separating the heated surface from the exterior cooled surface in this area. A two-temperature frequency regulation system will be applied, one to the outer wall, and the other to the cone, instead of movable tuners, to control the resonance. The coarse frequency will be set at manufacturing time, with the installation of small perturbers in the back wall.

The temperature distribution in combination with the vacuum (pressure) load was applied to the FEA model to determine the stress in the cavity and the distortion under load. The peak stress is less than $155 \mathrm{MPa}$ (a conservative $40 \%$ of the maximum yield strength of AISI 1020 steel) while the critical acceleration gap changes by a modest $0.17 \mathrm{~mm}(0.4 \%)$.

The beam exit hole and pipe diameter is presently set at $2.5 \mathrm{~cm}$. Such a pipe size is compatible with a geometry like the one used, for example, in the PITZ photoinjector [41]. In this kind of scheme, a $\sim 45 \mathrm{deg}$ mirror, located along the beam pipe downstream the gun, allows to direct the laser beam almost perpendicularly to the cathode surface. We are also evaluating the possibility of illuminating the cathode with a smaller angle of incidence respect to its plane (laser port on the exit wall of the cavity). The decision for the final configuration will account for the cathode performance as well as for other possible effects such as wakefields induced by the laser mirror [41, 42], or cavity field perturbation due to the laser ports.

\section{Beam Dynamics Simulations}

In order to control space charge effects in a similar manner to the approach taken for DC guns, the VHF gun is designed to deliver a relatively long electron bunch of several tens of picoseconds. This bunch is delivered to an injection system for additional acceleration, bunch compression, and emittance compensation/manipulation prior to further acceleration and injection into undulators.

We simulated the VHF gun performance by using the Advanced Photoinjector EXperiment (APEX) layout. APEX is a Lawrence Berkeley National Laboratory proposal for a beamline conceived to address fundamental issues in high average current, high brightness beam production for soft $\mathrm{x}$-ray FEL applications.

The APEX layout is schematically shown in Fig. 6. A UHF single-cell buncher, receives the $750 \mathrm{kV}$ beam 


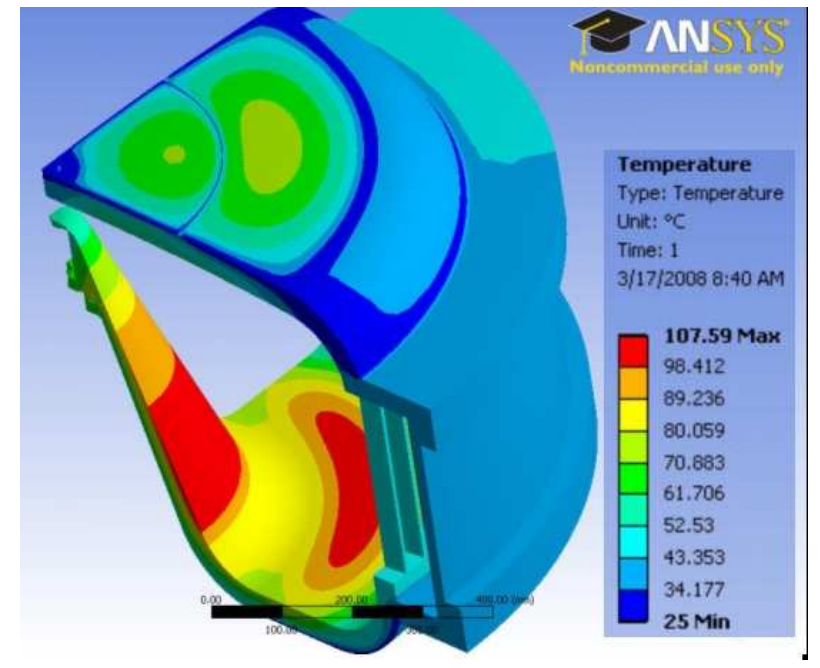

Figure 5:

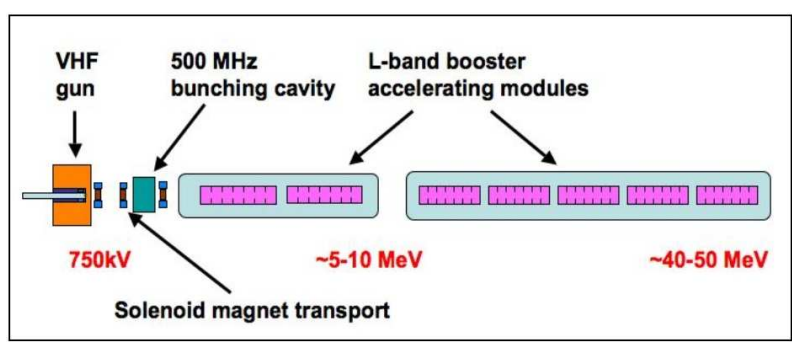

Figure 6:

from the VHF gun and introduces an energy chirp to allow compression of the beam. A L-band pre-booster then takes the mildly relativistic $750 \mathrm{kV}$ beam and accelerates it to the relativistic energy of $\sim 10 \mathrm{MeV}$, while completing the longitudinal compression and the matching of the transverse beam parameters necessary for the complete emittance compensation [43, 44]. The last Lband linac module finally accelerates the beam to the energy of $\sim 50 \mathrm{MeV}$. Solenoid magnets downstream the gun provide transverse focusing.

In the following paragraphs, we describe an example of the simulations we performed for characterizing the performance of the VHF gun. The example shows a solution that (although not completely optimized and based on some simplifying assumptions) substantiates the possibility of an injector using the VHF gun to generate a beam with parameters in the range required for operating an ERL or a FEL.

The ASTRA [45] code was used to model the performance of the RF gun and APEX, and the parameters at

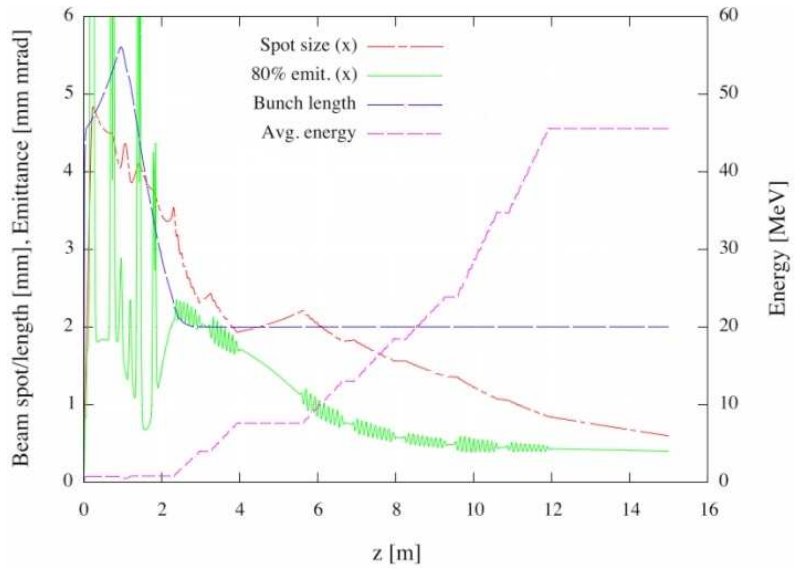

Figure 7:

the photocathode that were used as the input for the simulations are shown in Table 2. The thermal emittance of the emitted beam is taken to be very small to better study the effects of the space charge and the low frequency RF components on the evolution of beam quality in the photoinjector beamline.

The evolution of the main beam parameters is shown in Fig. 7, from the generation at the photocathode $(\mathrm{z}=$ $0 \mathrm{~m})$ to beyond the exit of the second linac module $(\mathrm{z}=$ $15 \mathrm{~m}$ ). At this point the beam has an average energy of slightly higher than $45 \mathrm{MeV}$ and a bunch length (duration) of $2 \mathrm{~mm}(\sim 6.7 \mathrm{ps}$ r.m.s. $)$. Emittance compensation has been carried out, with the final projected, normalized emittance of less than $0.5 \mathrm{~mm}$ mrad for $80 \%$ of the beam. The variation of the slice current and normalized emittance at the injector exit $(\mathrm{z}=15 \mathrm{~m})$ is shown in Fig. 8. After compression, the longitudinal profile takes on a skewed, pseudo-Gaussian distribution with a peak current of $\sim 32$ Amperes. The average value of the slice emittance (weighted by slice charge) is $\sim 0.35 \mathrm{~mm}$ mrad.

Even if not completely optimized, the results from this example show the capability of the VHF gun to operate in a scheme and in a regime compatible with the operation of a 2-2.5 GeV linac-based VUV-soft x-ray FEL or ERL. In fact, normalized emittances and bunch charges are in the required ranges, and the bunch length at the exit of the injector is comparable with that requested for an ERL [8]. For the FEL case, in order to achieve the desired peak currents, a further compression downstream in the accelerator is necessary. Simulations with lower and higher charge per bunch have been also performed showing comparable results. Of course, the final design of an injector based on the VHF gun re- 


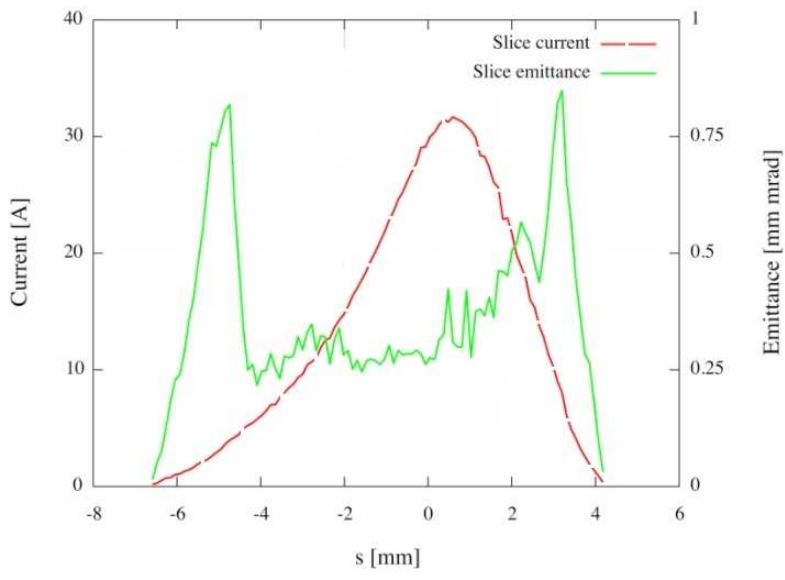

Figure 8:

quires simulations using a more realistic cathode thermal emittance (once the choice on the cathode type is finalized), and an accurate optimization of all the injector parameters similar to the one performed at Cornell for their DC gun-based injector [8].

The cathode choice and the related gun repetition rate are deeply connected to the available laser technology. Present pulsed fiber lasers are capable of $\sim 200 \mathrm{~W}$ of average power in the infrared [46]. For example, a $\sim 20 \mathrm{~W}$ average power laser can drive high quantum efficiency semiconductor cathodes directly (GaAs) or by higher harmonic conversion (CsTe) to generate $\mathrm{nC}$ bunches at $1 \mathrm{MHz}$ repetition rate. A $200 \mathrm{~W}$ laser after higher harmonic conversion, could be used instead for operating with low quantum efficiency metal cathodes $(\mathrm{Cu}$ and $\mathrm{Mg}$ for example) to $\sim 100 \mathrm{kHz}$ repetition rates with $\mathrm{nC}$ bunches. Considerably higher repetition rates can be obtained if the charge per bunch is reduced.

We have also performed initial optimization studies of some of the VHF gun parameters. In particular, we were interested in studying the effects on the beam quality at the injector high-energy exit due to variations in the geometry of the electrodes that comprise the accelerating gap, and to variations of the cavity resonant frequency [21]. The simulations showed that for high charge bunches, the overall beam quality after longitudinal compression and emittance compensation can be remarkably insensitive to the details of the gap geometry. In this situation, a flat cathode with maximum accelerating gradient is thus favored over more complicated geometries. Additional studies are required for understanding the effects of the cathode area geometry in the low charge (hundred $\mathrm{pC}$ ) regime.

Simulations also showed that beam dynamics and beamline optimization are marginally affected by RF gun frequency variation over a broad band (from 50 to $200 \mathrm{MHz}$ ). This allowed us to define the resonant frequency for the gun based only on RF and mechanical considerations. The $106 \mathrm{MHz}$ is one of the possible choices trading between a lower power density on the cavity walls (for an easy cooling of the structure) and a reasonably high voltage breakdown limit, between a practical size and sufficiently large apertures for an efficient pumping system, between cost of the cavity and cost of the RF power source [47]. We are also evaluating and designing $200 \mathrm{MHz}$ and $352 \mathrm{MHz}$ versions of the cavity in order to compare these different tradeoffs within the VHF bandwidth.

\section{Conclusions}

In summary, we have presented the concepts and the preliminary design results for a high brightness $\mathrm{CW}$ normal-conducting RF gun operating in the VHF frequency range. Beam dynamics simulations showed that the quality of the beam generated by such a source is suitable for ERL and high average power FEL applications. The simple and reliable scheme based on mature RF and mechanical technology makes the VHF gun an appealing alternative to DC and super-conducting electron guns when high repetition rates are required.

We acknowledge contributions of J. M. Byrd, G. Huang, D. Li, E. Parodi, A. Ratti, W. Waldron, R. Wilcox and A. Zholents. We also thanks J. DeFord for the multipactoring simulations with Analyst ${ }^{T M}$.

This work was supported by the Director of the Office of Science of the U.S. Department of Energy under Contract No. DE-AC02-05CH11231.

\section{References}

[1] J. Corlett, et al., Proc. of 2007 IEEE Part. Accel. Conf., Albuquerque, NM, USA, 2007, 11671169.

[2] D. Kramer, E. Jaeschke, W. Eberhardt (Editors), The Bessy Soft X-Ray Free Electron Laser, ISBN 3-9809534-0-8, 2004

[3] K.-J. Kim, Y. Shvyd'ko, S. Reiche, Phys. Rev. Lett. 100 (2008) 244802

[4] A. M. M. Todd, State-of-the art electron guns and injector designs, Presented at the 32nd ICFA Advanced Beam Dynamics Workshop on Energy Recovery Linacs, Newport News, VA, USA, March 19, 2005. To be published on JACoW (http://accelconf.web.cern.ch/accelconf/).

[5] A. M. M. Todd, Nuclear Inst. and Methods in Physics Research A 557 (2006) 3644.

[6] I. Ben-Zvi, I.V.Bazarov, Nuclear Inst. and Methods in Physics Research A 557 (2006) 337344.

[7] Summary of working group on guns and injectors, 41st Advanced ICFA Beam Dynamics Workshop on Energy Recovery Linacs, Daresbury Laboratory, U.K., May 21-25, 2007. 
[8] I. Bazarov, C. Sinclair, Phys. Rev. ST Accel. Beams 8 (2005) 034202 .

[9] C. Hernandez-Garcia, et al., Proc. of 26th International Free Electron Laser Conference, Trieste, Italy, 2004, 558560.

[10] B. M. Dunham, et al., Proc. of 2007 IEEE Part. Accel. Conf., Albuquerque, NM, USA, 2007, 12241226.

[11] A. J. Teichert, et al., Proc. of the 27th International Free Electron Laser Conference, Stanford, CA, USA, 2005, 534536.

[12] A. M. M. Todd, et al., Proc. of 2005 IEEE Part. Accel. Conf., Knoxville, TN, USA, 2005, 22922294.

[13] R. Brinkmann, Y. Derbenev, K. Flottmann, Phys. Rev. ST-Accel. Beams 4 (2001) 053501.

[14] P. Emma, et al., Phys. Rev. ST-Accel. Beams 9 (2006) 100702.

[15] J . W. Staples, S. P. Virostek and S. M. Lidia, Proc. of the 2004 European Particle Accelerator Conference, Lucerne, Switzerland, July 2004, 473475.

[16] D. Dowell, et al., Appl. Phys. Lett. 63 (1993) 20352037.

[17] R. A. Rimmer, Proceedings of the 2005 Particle Accelerator Conference, Knoxville, TN USA, May 2005, 30493051.

[18] S.S. Kurennoy, et al., Nuclear Inst. and Methods in Physics Research A 528 (2004) 392396.

[19] S.S. Kurennoy, et al., Proc. of 2005 IEEE Part. Accel. Conf., Knoxville, TN, USA, 2005, 28662868.

[20] J. Staples, F. Sannibale, S. Virostek VHF-band Photoinjector, Lawrence Berkeley National Laboratory CBP Tech Note-366, October 2006.

[21] Steven Lidia, et al., Development of a high-brightness VHF electron source at LBNL, ERL07 Workshop, Daresbury, U.K., May 2007. To be published.

[22] J. Staples, et al., Proc. of 2007 IEEE Part. Accel. Conf., Albuquerque, NM, USA, 2007, 29902992.

[23] J. Corlett, et al., ICFA Beam Dynamics Newsletter 42 (2007) 51 58.

[24] R. Dei-cas, et al., Nuclear Inst. and Methods in Physics Research A 296 (1990) 209216.

[25] S. Joly et al., Nuclear Inst. and Methods in Physics Research A 331 (1993) 199203.

[26] J.-G. Marmouget, et al., Proc. of the 2002 European Particle Accelerator Conference, Paris, France, June 2002, 17951797.

[27] D. Guilhem, et al., Proc. of the 2006 European Particle Accelerator Conference, Edinburgh, Scotland, July 2006, 19271929.

[28] H. Ino, et al., Proc. of the XX International Linac Conference, Monterey, CA USA, August 2000, 10151017.

[29] T. Abe, et al., Proc. of the 2006 European Particle Accelerator Conference, Edinburgh, Scotland, July 2006, 13071309.

[30] http://www.jlab.org/ genfa/fishpact/

[31] Analyst ${ }^{T M}$ code, http://www.staarinc.com.

[32] J. Staples, Multipactoring Calculations for the VHF Photoinjector Cavity using Fishpact, Lawrence Berkeley National Laboratory CBP Tech Note-377, July 2007.

[33] P. Michelato, D. Sertore, C. Gesmundo High quantum efficiency photocathode preparation system for the TTF injector II, Proceedings of 21st Free-Electron Laser Conference (FEL'99), Hamburg, Germany, August 23-26, 1999. Eds. J. Feldhaus, H. Weise. Elsevier Science B.V., (2000)

[34] D. Sertore, et al., Nuclear Inst. and Methods in Physics Research A 445 (2000) 422426.

[35] J. Staples, G. Huang, R. Wells, Pump Port Calculations for the VHF Photoinjector Cavity, Lawrence Berkeley National Laboratory CBP Tech Note-378, July 2007.

[36] J. Staples, VHF Injector Pumping Slot RF Shielding Effectiveness, Lawrence Berkeley National Laboratory CBP Tech Note379, August 2007.

[37] MAFIA, CST GmbH., Bad Nauheimer Strasse 19, 64289 Darmstadt, Frankfurt, Germany (http://www.cst.com/).
[38] http://www.saesgetters.com

[39] http://laacg1.lanl.gov/laacg/services/download_sf.phtml.

[40] http://www.ansys.com.

[41] S. Setzer, et al., Proc. of the 2004 European Particle Accelerator Conference, Lucerne, Switzerland, July 2004, 19841986.

[42] E. Arevalo, et al., Proc. of the 2008 European Particle Accelerator Conference, Genova, Italy, June 2008, 217219.

[43] B.E. Carlsten, Nuclear Inst. and Methods in Physics Research A 285 (1989) 313319.

[44] L. Serafini, J.B. Rosenzweig, Physical Review E 55 (1997) 7565 7590 .

[45] http://www.desy.de/ mpyflo/Astra_dokumentation.

[46] C. Brooks and F. Di Teodoro, Appl. Phys. Lett. 89 (2006) 111119-1 111119-3.

[47] J. Corlett, et al., Choice of a VHF cavity design for a highbrightness, high repetition rate electron gun, Lawrence Berkeley National Laboratory CBP Tech Note-384, December 2007. 
Figure 1. 3-D CAD model of the normal conducting VHF cavity for a high repetition rate photoinjector. Slots around the periphery allow for vacuum pumps located in the antechamber. (In color online)

Figure 2. Possible multipactoring modes vs. accelerating gradient for the VHF structure. Fishpact calculation. (In color online)

Figure 3. Cathode area detail. (In color online)

Figure 4. Logarithm of the magnitude of the electric field in the outer pump slot region of cavity. (In color online)

Figure 5. Temperature distribution on the cavity calculated at the nominal power. (In color online)

Figure 6. The Advanced Photoinjector EXperiment (APEX) schematic layout. APEX was used as a bench test for simulating the performance of VHF gun described in this paper. (In color online)

Figure 7. Example of beam quality versus the longitudinal position along APEX. (In color online)

Figure 8. Example of beam longitudinal distribution and slice emittance at the exit of APEX. (In color online)

\begin{tabular}{|l|r|c|}
\hline \multicolumn{1}{|c|}{ Parameter } & Value & Units \\
\hline Mechanical: & 56 & $\mathrm{~cm}$ \\
Cavity radius & 58 & $\mathrm{~cm}$ \\
Total length & 4 & $\mathrm{~cm}$ \\
Accelerating gap & 0.17 & $\mathrm{~mm}$ \\
Max gap deformation & & \\
(combined thermal and pressure load) & 156 & $\mathrm{MPa}$ \\
Max von-Mises stress & 83 & ${ }^{\circ} \mathrm{C}$ \\
Peak temperature rise & 106 & $\mathrm{MHz}$ \\
Electrical: & 37800 & \\
Frequency & $\mathrm{CW}$ & \\
Q0 & 750 & $\mathrm{kV}$ \\
Repetition Rate & 20 & $\mathrm{MV} / \mathrm{m}$ \\
Gap Voltage & 26 & $\mathrm{MV} / \mathrm{m}$ \\
Field at cathode & 73 & $\mathrm{~kW}$ \\
Peak surface field & 8 & $\mathrm{~W} / \mathrm{cm}{ }^{2}$ \\
RF power for 750 kV & 4.1 & $\mathrm{~J}$ \\
Peak wall power density & & \\
Stored energy & $8.5 \times 10^{-11}$ & $\mathrm{Torr}$ \\
Vacuum: & 36 & \\
Pressure & 36 & \\
Number of vacuum pumping slots & $14-21$ & $\mathrm{MV} / \mathrm{m}$ \\
Number of getter pump modules &
\end{tabular}

Table 1: VHF gun nominal values for the main parameters

\begin{tabular}{|c|c|c|}
\hline Bunch charge & 500 & $\mathrm{pC}$ \\
\hline Long. distribution & Flat top & \\
\hline Bunch Length (FWHM) & 75 & $\mathrm{ps}$ \\
\hline r.m.s. energy spread & 0.1 & $\mathrm{eV}$ \\
\hline Transv. distribution & Parabolic & \\
\hline Spot size (r.m.s.) & 0.5 & $\mathrm{~mm}$ \\
\hline Thermal emittance & 0.05 & $\mathrm{~mm}-\mathrm{mrad}$ \\
\hline
\end{tabular}

Table 2: Beam parameters at the cathode used in the simulation 